\title{
The utility of the implementation science framework "Integrated Promoting Action on Research Implementation in Health Services" (i-PARIHS) and the facilitator role for introducing patient-reported outcome measures (PROMs) in a medical oncology outpatient department
}

\author{
Natasha A. Roberts ${ }^{1,2} \cdot$ Monika Janda ${ }^{1,3} \cdot$ Angela M. Stover $^{4,5} \cdot$ Kimberly E. Alexander $^{1} \cdot$ David Wyld $^{2,6}$. \\ Alison Mudge ${ }^{2,6}$ on behalf of the ISOQOL PROMs/PREMs in Clinical Practice Implementation Science Work Group
}

Accepted: 3 October 2020 / Published online: 21 October 2020

(c) The Author(s) 2020

\begin{abstract}
Purpose We evaluated the utility of the implementation science framework "Integrated Promoting Action on Research Implementation in Health Services" (i-PARIHS) for introducing patient-reported outcome measures (PROMs) into a medical oncology outpatient department. The i-PARIHS framework identifies four core constructs for implementation, including Facilitation, Innovation, Context and Recipients.

Methods A pilot study used the i-PARIHS framework to identify PROM implementation barriers and enablers to inform facilitation support strategies, such as training clinicians and staff, workflow support, technical support and audit and feedback. Pre- and post-implementation surveys were completed by 83 and 72 staff, respectively, (nurses, doctors and allied health), to assess perceived knowledge, enablers, barriers and utility of PROMs; and acceptability of the PROM intervention was also assessed post-implementation.

Results Important barriers included time constraints and previous experiences with technology. Enablers included good leadership support and a culture of learning. Facilitation strategies were used to overcome barriers identified in the i-PARIHS core domains. Compared to before the intervention, staff surveys showed improvement in perceived usefulness, perceived understanding and interpretation skills for PROMs. Staff perceptions about lack of time to use PROMs during visits remained a major perceived barrier post-implementation.

Conclusion The i-PARIHS framework was useful for guiding the implementation of PROMs in routine oncology care. The four core i-PARIHS constructs (Facilitation, Innovation, Context and Recipients) identified factors that directly impacted implementation, with Facilitation having a particularly important role to overcome these barriers. Oncology clinics and health systems considering implementing PROMs should consider having a dedicated Facilitator available during PROM implementation.
\end{abstract}

Keywords Patient-reported outcome measures $\cdot$ Quality of life $\cdot$ Implementation science $\cdot$ Clinical practice $\cdot$ Routine care

Natasha A. Roberts

natasha.roberts@health.qld.gov.au

1 Queensland University of Technology, Kelvin Grove, QLD, Australia

2 Royal Brisbane and Women's Hospital, Herston, QLD, Australia

3 Centre for Health Services Research, The University of Queensland, Woolloongabba, QLD, Australia
4 Department of Health Policy and Management, University of North Carolina at Chapel Hill, Chapel Hill, NC, USA

5 Lineberger Comprehensive Cancer Center, University of North Carolina at Chapel Hill, Chapel Hill, NC, USA

6 School of Clinical Medicine, University of Queensland, St Lucia, QLD, Australia 


\section{Background}

In clinical trials, patient-reported outcome measures (PROMs) improve communication between patients and clinicians, resulting in quality of life and survival benefits [1-3]. Implementation strategies are key to translating health innovations into practice [4], but little is known about the optimal implementation strategies for PROM integration in medical oncology outpatient departments.

Care delivery transformation within the clinical environment of an outpatient oncology clinic is complex [5-7]. This complexity comes from the many levels of the larger health ecosystem [8] into which outpatient oncology wards are embedded [9], as well as the diverse needs of cancer patients undergoing a multitude of treatment protocols and follow-up [10]. Teams of multidisciplinary health professionals may be involved in each patient's care and they may have competing priorities with diverse knowledge, attitudes and beliefs [11, 12]. In randomised controlled trials (RCTs) testing the value of PROMs, the clinical trial coordinator often played an invisible role facilitating uptake of the PROM innovation with patients and staff teams (e.g. conducting training sessions and running clinic-level reports) $[13,14]$. However, in routine clinical practice, there may not be investment in a dedicated implementation support role to support this complex process [15]. To implement a new intervention into this type of an environment requires insight into the interplay of the innovation (PROMs), the context and setting and the people and roles involved [16].

The multiple stakeholders required to implement PROMs include patients, families, nursing staff, administrative staff, medical staff, allied health professionals and records managers, all need to be engaged in using PROMs [5]. Their involvement is required to ensure initiating, handling, interpreting, and responding to PROMs as part of routine care delivery $[17,18]$, thus making this a complex healthcare intervention [19].

We used the implementation science framework "Integrated Promoting Action on Research Implementation in Health Services" (i-PARIHS) to examine factors impacting the use of the PROM version of the Common Terminology Criteria for Adverse Events (PRO-CTCAE) [20] during patient care visits.

i-PARIHS is a framework specifically designed for implementing complex interventions in healthcare [21] and is unique in emphasising the role of a Facilitator as the central construct of successful implementation. Like other determinant frameworks in implementation science [22], i-PARIHS can be used to help understand and overcome the multiple influences impacting implementation. Based strongly on experiential learning and empirical evidence, whilst drawing on a range of behaviour change theories, i-PARIHS can be used to plan, guide and understand complex implementation in healthcare [23]. This paper reports on a pilot implementation case study using the constructs of i-PARIHS ( see Box 1).

The aims of this paper are to

1. describe this case study of PROMs implementation in routine oncology practice using the i-PARIHS framework [21], to better understand what conditions are critical to enable integration of this intervention into the clinic;

2. describe the Facilitator role and how it was used to overcome barriers to PROM implementation;

3. determine changes in clinician and staff perceptions of acceptability [4] and knowledge of PROMs, and perceived skills in interpreting and using PROMs with patients and

4. summarise lessons learned to inform implementation, sustainability, scale and spread of future PROM projects.

\section{Box 1: The intervention design}

The PROMs intervention consisted of patients completing the PRO-CTCAE in the waiting room of two oncology clinics in Australia, and clinicians reviewing patient responses and acting on burdensome symptoms. The core-set PRO-CTCAE measures cancer-specific symptoms such as constipation, diarrhoea, nausea, vomiting, shortness of breath, fatigue, anxiety, low mood, pain, peripheral neuropathy, and other symptoms [9]. Patients completed the PRO-CTCAE items via a touchscreen workstation in the waiting room. A paper copy of the patient's PROM report was automatically printed, then immediately available to the care team for use during the visit. The PROM report was also imported into the patient's scanned electronic medical record for future reference. The immediate availability of standardised symptom reports has been shown in RCTs to improve symptom detection, more consistent management of symptoms, quality of life and mortality [13].

Implementation took place in two oncology outpatient clinics in a large metropolitan hospital in Queensland, Australia. This hospital provides oncology services for the metropolitan area as well as specialist referral services for patients across the state. 


\section{Methods}

\section{Implementation framework}

To comprehensively understand PROM implementation issues presented in the literature [25-29], and to inform selection of an implementation framework, we previously undertook a scoping review of systematic reviews [19]. This review identified key themes of (1) engaging clinicians, (2) integration of technology for PROMs and (3) strategies to respond to PROMs data [14]. It was identified that these themes correspond to core constructs of the i-PARIHS framework (Recipients, Innovation and Context), and so it was chosen as the guiding framework for our pilot study [30]. In this report, we present i-PARIHS terms in italics.

Each core construct of i-PARIHS has a sub-set of potential factors that can become enablers or barriers for implementation that can be addressed using Active Facilitation, which has been demonstrated to increase uptake of research evidence in primary care $[24,31,32]$ and is "how" the implementation happens. Central to the i-PARIHS framework, Facilitation is "the construct that activates implementation through assessing and responding to characteristics of the Innovation and the Recipients (both as individuals and in teams) within their Contextual setting" [21, p. 6].

For this study, an internal novice Facilitator (NR), an oncology nurse experienced as a clinical trial coordinator, conducted study activities pre-, during- and post-implementation. Consistent with the concept of the Facilitator's journey [30], the novice Facilitator engaged with a expert Facilitator (AM) and a local multidisciplinary peer group of clinician-implementers for reflection (and peer learning) every 1-2 months during the implementation. She also had structured academic support from a research team with university academic affiliations to the setting.

The scoping review of systematic reviews [14] theme of engaging with clinicians aligned with the i-PARIHS Recipients construct of "who" receives the intervention. Whilst the Recipients included clinicians, patients and health administration staff, this case study focuses on the views of the clinical staff, as the group who reviewed the PROM, interpreted patients' responses and responded to achieve the anticipated clinical outcomes of the intervention. Clinical staff in the setting included nurses, doctors (specialist oncologists and trainees) and a range of allied health professionals, including dietitians, physiotherapists, occupational therapists, speech therapists, psychologists and social workers.

Within the i-PARIHS framework, potential Recipient enablers and barriers include factors, such as motivation, value \& beliefs, clinical consensus, local opinion leaders, existing data sources, skills and knowledge, time and resources, learning environment, collaboration and teamwork, power and authority and professional boundaries and networks [21].

In the scoping review of systematic reviews [14], the theme of technology integration for PROMs aligned with i-PARIHS's construct of the Innovation. This is "what" is implemented and is described in more detail in Box 1 . Potential barriers and enablers within the Innovation construct include underlying knowledge sources, clarity, degree of fit (compatibility or contestability), degree of novelty, likely boundaries, trialability and relative advantage [21].

The scoping review of systematic reviews theme [14] of strategies to respond to PROMs data aligned with i-PARIHS's Context, "where" the implementation takes place and encompasses the characteristics of the setting.

Enablers and barriers considered under the Context domain included characteristics of the inner and outer context. Inner Context factors included formal and informal leadership support, culture, past experience of change, mechanisms for embedding change and learning and evaluation and feedback processes. Outer Context factors included organisational priorities, structure, leadership and senior management support, systems and processes, culture, history of innovation and change, and absorptive capacity (the ability to absorb change in the Context) [21].

\section{Study design}

This study used a descriptive mixed methods case study design nested within a prospective trial of PROMs implementation in two oncology clinics [33, 34]. The research protocol of the trial is presented elsewhere [35].

\section{Data collection and analysis}

Qualitative data were coded by the Facilitator (NR) [35] using a content analysis [36], informed by the i-PARIHS framework [21]. Data collected by the Facilitator over a 12-month period was informed by the research protocol. These data included field notes comprising structured observations of activity for context assessments and process mapping [21], weekly case report forms and transcribed quotes from Recipients as memos. Results from the pre-implementation findings had been previously shared (and amended for accuracy) with Recipients to plan the implementation and during the iterative evaluation cycles, then also reviewed by staff post-implementation. Additionally, free text responses on questions about perceived knowledge, perceived usefulness and perceived enablers and barriers in a clinician survey completed pre- and post-implementation were included as a qualitative dataset [35]. These qualitative data were collated for the evaluation by NR, and informed discussions with an expert Facilitator (AM). 
To determine if each factor was a barrier or enabler from the Facilitator perspective, the novice Facilitator (NR) and expert Facilitator (AM) separately identified and rated factors along a visual continuum of barrier to enabler, and then combined their ratings by consensus. Consensus discussions were summarised into templated tables, and illustrative examples of facilitation strategies were agreed on by discussion. Final tables were reviewed for face validity by a third rater, the lead senior academic team member not directly involved in the health service (MJ). Whilst we recognise that such judgements are inherently subjective, potential biases on the part of the Facilitator (NR) were thus mitigated through a number of mechanisms, including the cycles of evaluation with participants, reflexive monitoring, expert facilitation discussions and collaboration with a wider academic team.

Quantitative data included a survey adapted from Rouette et al. [35, 37] used to elicit clinician and staff perceptions of PROM acceptability [34], knowledge and utility of
PROMs, and enablers and barriers. Surveys were distributed to nursing, medical and allied health professionals involved in providing care to patients, before and after implementing the intervention. Data were summarised by discipline group, reporting the proportion of respondents who agreed or strongly agreed with each statement and are presented in a table for comparison.

The potential conflict of interest that could come from the novice Facilitator's relationship with participants was addressed in the design of the study, including the ethical considerations that were integrated into the study protocols. For the anonymous staff surveys, participation was considered consent and staff could decline participation in surveys simply by not completing them. During the implementation phase, staff in-service education was provided, marketing flyers distributed and information sheets provided. These resources explicitly stated that participation was voluntary and staff could opt out at any time in writing on case report

Table 1 Consensus expert and novice facilitator ratings, with examples, of i-PARIHS constructs in the Recipients domain as a barrier or enabler

\begin{tabular}{|c|c|c|c|c|}
\hline \multirow[t]{2}{*}{ i-PARIHS recipient constructs } & \multicolumn{3}{|l|}{ Rating } & \multirow[t]{2}{*}{ Example } \\
\hline & Barrier & & Enabler & \\
\hline Motivation & & $\mathrm{X}$ & & Variation in perceived utility of PROMs within and between disciplines \\
\hline Values/beliefs & & & $\mathrm{X}$ & Strong professional values of person-centred care amongst staff groups \\
\hline Local opinion leaders & & & $\mathrm{X}$ & Over the course of the project, peer champions for PROMs emerged \\
\hline Existing data sources & $\mathrm{X}$ & & & Lack of local data/reporting processes for patient symptoms \\
\hline Skills and knowledge & & $\mathrm{X}$ & & Variable knowledge of PROMs within and between disciplines \\
\hline Time and resources & $\mathrm{X}$ & & & Staff feeling pressured with competing tasks and priorities \\
\hline Collaboration and teamwork & $\mathrm{X}$ & & & Professional silos and discipline-specific work practices \\
\hline Power and authority & & & $\mathrm{X}$ & Project support by Medical Director as co-investigator \\
\hline Professional boundaries and networks & $X$ & & & $\begin{array}{l}\text { Limited communication between disciplines, expressed concerns that } \\
\text { other disciplines may not engage leading to disproportionate burden } \\
\text { on some staff }\end{array}$ \\
\hline
\end{tabular}

Table 2 Consensus expert and novice facilitator ratings, with examples, of i-PARIHS constructs in the Innovation construct were a barrier or enabler

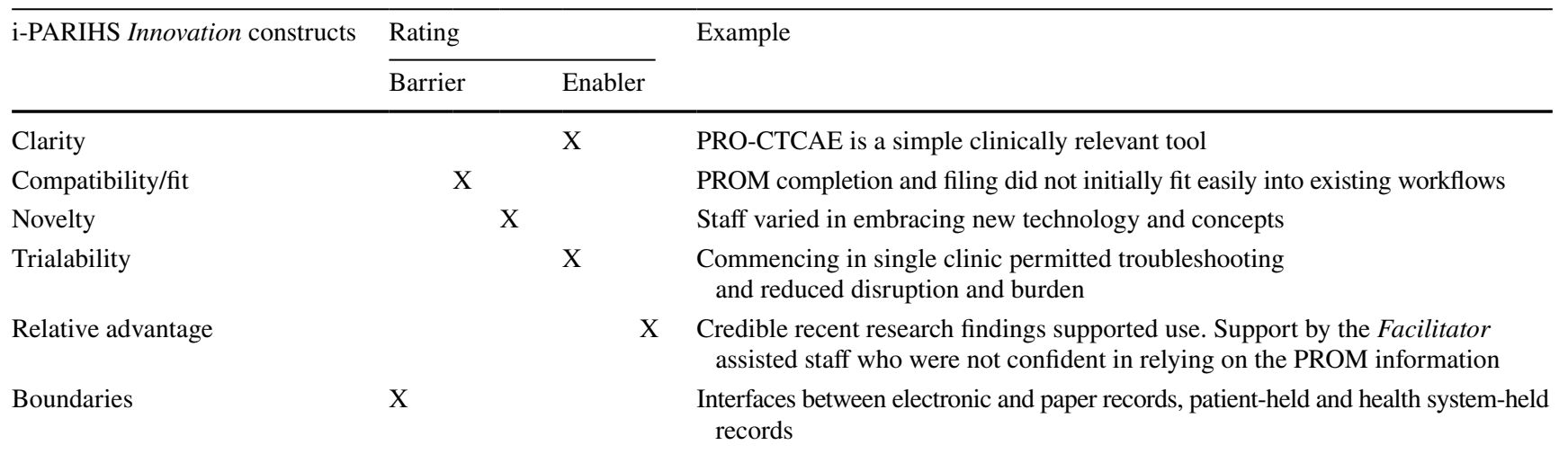


Table 3 Consensus expert and novice facilitator ratings, with examples, of whether i-PARIHS constructs in the Context as a barrier or enabler

\begin{tabular}{|c|c|c|c|c|}
\hline \multirow[t]{2}{*}{ i-PARIHS Context constructs } & \multicolumn{3}{|l|}{ Rating } & \multirow[t]{2}{*}{ Example } \\
\hline & Enabler & & Enabler & \\
\hline Formal and informal leadership networks & & & $\mathrm{X}$ & $\begin{array}{l}\text { Project lead had credibility and moderate support from medical and } \\
\text { nursing leadership and peers }\end{array}$ \\
\hline Culture & & $\mathrm{X}$ & & $\begin{array}{l}\text { Competing priorities of throughput and patient-centred care leading to } \\
\text { staff tension and competing priorities }\end{array}$ \\
\hline Past experience with change & $\mathrm{X}$ & & & $\begin{array}{l}\text { Staff had experience with previous unsuccessful initiatives that became } \\
\text { burdensome }\end{array}$ \\
\hline Mechanisms for embedding change & $\mathrm{X}$ & & & Previous changes had often failed to be sustained \\
\hline Evaluation and feedback processes & & & $\mathrm{X}$ & $\begin{array}{l}\text { Staff had some familiarity with audit and feedback, e.g. safety and qual- } \\
\text { ity audits }\end{array}$ \\
\hline Learning environment & & & $\mathrm{X}$ & $\begin{array}{l}\text { Available in-service education opportunities for staff; strong emphasis } \\
\text { on research evidence }\end{array}$ \\
\hline Organisational priorities & & $\mathrm{X}$ & & Efficiency and risk were highlighted as important \\
\hline Leadership and senior management support & & $\mathrm{X}$ & & $\begin{array}{l}\text { Research funding provided some credibility and funding discretion, but } \\
\text { not seen as core activity }\end{array}$ \\
\hline Structure and systems & & $\mathrm{X}$ & & $\begin{array}{l}\text { Large oncology service; disciplines have separate reporting; complex } \\
\text { interface with existing record systems }\end{array}$ \\
\hline Absorptive capacity & & & $\mathrm{X}$ & $\begin{array}{l}\text { Staff were accustomed to accommodating practice changes (e.g. fre- } \\
\text { quent updates to medical protocols) often through "workarounds" }\end{array}$ \\
\hline Learning networks & $\mathrm{X}$ & & & $\begin{array}{l}\text { Limited connections with other services that were successfully using } \\
\text { PROMs }\end{array}$ \\
\hline
\end{tabular}

forms, or by simply not participating in the implementation work.

\section{Results}

\section{Barrier and enabler ratings from the Facilitator perspective}

Tables 1, 2, 3 show the consensus ratings of the novice and expert Facilitators, addressed for each i-PARIHS construct. Strategies used to overcome barriers are discussed in the text.

Features of the Recipients (focussing on clinical staff) are summarised in Table 1. The Facilitator used educational strategies (e.g. training sessions with clinicians and staff) to introduce PROMs to clinicians and staff and how to interpret patient responses. An identified barrier was a concern that colleagues would not engage with PROMs, which could lead to disproportionate burden on some staff or even failure of the intervention. The Facilitator used open dialogue to alleviate these concerns.

Table 2 describes the Innovation domain. The trialability (the ability to test the Innovation at a small scale) improved the degree of fit (how well the Innovation integrated into workflows), by being flexible and using iterative implementation, with the Facilitator supporting each step of the process (e.g. patient report completion, report generation, filing and interpretation) through hands-on technical support and contingent problem solving. Through consultation with all disciplines as well as "hands-on" contingent assistance, the Facilitator was able to model and teach these changes as the implementation progressed. The PROMs intervention was reported by staff on surveys as having relative advantage.

Table 3 summarises features of the local and organisational Context that influenced implementation. Staff said they were concerned that the health service would not have the resources to respond to additional patient needs identified through the PROM information. The Facilitator needed to maintain momentum by intermittently providing PROMs reports at clinic level, and ensuring they were consistently transferred into the EMR record, until the workflows were established to ensure staff confidence. It took much longer than anticipated for PROMs workflows to become independent from the Facilitator. Audit and feedback of the implementation included routine reporting the proportions of PROMs completed by patients and PROMs acknowledged by staff at team meetings of staff [35].

\section{Clinician and staff perspectives of barriers and enablers}

Ninety surveys were distributed to medical, allied health and nursing professionals currently working in the outpatient clinic who attended staff meetings or scheduled 
Table 4 Demographic characteristics of staff survey participants

\begin{tabular}{lll}
\hline Staff participant & $\begin{array}{l}\text { Pre-implementation } \\
N=83\end{array}$ & $\begin{array}{l}\text { Post-implementation } \\
N=72\end{array}$ \\
\hline $\begin{array}{l}\text { Demographics } \\
\text { Gender: }\end{array}$ & \\
Male & $19(23 \%)$ & $14(19 \%)$ \\
Female & $64(77 \%)$ & $58(81 \%)$ \\
Age & & \\
20-40 years & $24(29 \%)$ & $30(42 \%)$ \\
40-60 years & $47(57 \%)$ & $42(58 \%)$ \\
$<60$ years & $12(14 \%)$ & $0(0 \%)$ \\
Clinician group & & \\
Nursing & $53(64 \%)$ & $42(58 \%)$ \\
Medical & $19(23 \%)$ & $20(28 \%)$ \\
Allied health & $11(13 \%)$ & $10(14 \%)$ \\
\hline
\end{tabular}

in-service education, during a 4-week period, pre-implementation and following implementation, with 83 and 72 responses received, respectively, at these time points. The demographic characteristics of participants are presented in Table 4.

Post-implementation, perceived knowledge and understanding of PROMs in clinical practice increased. For example, pre-intervention $82 \%$ of doctors, $85 \%$ of allied health professionals and $0 \%$ of nurses reported good/very good understanding of PROMs, and $41 \%$ of doctors, $77 \%$ of allied health professionals and $2 \%$ of nurses reported good/very good PROM interpretation skills. Post-intervention, $95 \%$ of doctors, $70 \%$ of allied health professionals and $48 \%$ of nurses reported good or very good understanding of PROMs, and $85 \%$ of doctors, $50 \%$ of allied health professionals and $48 \%$ of nurses reported good/very good interpretation skills. Although more staff agreed that the PROM reports were useful and intended to use them in practice following implementation, lack of time remained a barrier to routine use from the nursing and medical perspective. Table 5 shows the pre- and post-implementation responses for each survey item.

\section{Discussion}

This study evaluated implementing PROMs into the routine care of an oncology outpatients' department using the i-PARIHS framework. The i-PARIHS domains of Facilitation, Innovation, Recipients and Context were useful for identifying barriers and enablers so that tailored strategies could be developed and put in place. The use of an implementation science framework in a research study offered new insights into the characteristics of implementation in differing Contexts and Recipients [16]. Using i-PARIHS provided a systematic way to organise observations, provide new insights into how research findings can be translated and how they impact delivery of care in routine oncology health services $[16,38,39]$. This can inform future larger studies, which in turn can be used to improve outcomes in large populations of patients [40].

By using i-PARIHS, we were able to identify, measure and report on how Facilitation ameliorated barriers to implementation. Core Facilitation strategies included education, awareness raising, and audit and feedback [41-43]. Facilitation took a dynamic and flexible approach balanced with the formal structure of audit and feedback that acted to inform and regularly engage with Recipients (care teams). Similarly, in previous work, Harvey et al. [40] in the FIRE study found that regular meetings were important for implementation to offer goal-focussed practical support, technical assistance and interactive problem solving.

Facilitation is a recognised implementation strategy [43], which uses a relational approach to problem solving and may involve trained personnel external to the healthcare context [40]. In our study, the novice Facilitator was part of the local staff, but was supported by an experienced Facilitator and team to identify and reflect on appropriate strategies [21]. The support of an experienced Facilitator was valuable whilst the novice Facilitator developed increased awareness, skills and knowledge. Besides providing verbal guidance and problem solving, the novice Facilitator had to be physically present in the clinic to support the clinicians, problem solve

Table 5 Percentage of staff participants endorsing survey questions before and after implementation

\begin{tabular}{|c|c|c|}
\hline Survey question & Pre $(n=83), n(\%)$ & Post $(n=72), n(\%)$ \\
\hline My understanding of the concept of PROs in clinical practice is very good to fair (vs poor to very poor) & $25(30)$ & $46(64)$ \\
\hline $\begin{array}{l}\text { In terms of interpreting PROs, I feel my interpretations skills are very good to fair (vs poor to very } \\
\text { poor) }\end{array}$ & $12(14)$ & $42(58)$ \\
\hline $\begin{array}{l}\text { I generally perceive PROs as being very useful to somewhat useful (vs a little useful to I don't know } \\
\text { enough to have an opinion) }\end{array}$ & $38(46)$ & $52(72)$ \\
\hline $\begin{array}{l}\text { I feel my that my colleagues generally perceive PROs as very useful to somewhat useful (vs a little use- } \\
\text { ful to I don't know enough to have an opinion) }\end{array}$ & $35(42)$ & $33(46)$ \\
\hline $\begin{array}{l}\text { A lack of time would be a barrier to discussing PRO outcomes with my patients in clinic all of the time } \\
\text { to most of the time (vs sometimes to none of the time) }\end{array}$ & $58(70)$ & $43(60)$ \\
\hline
\end{tabular}


to make the PROM intervention fit the Context and maintain implementation momentum. This demand on the Facilitator illustrates the tension between a Facilitator doing the work rather than enabling implementation [43]. By keeping up momentum through workflows, the Facilitator enabled the experience of using a PROM in clinical practice for staff $[44,45]$. However, for sustainability, the intervention would need to be fully integrated into routine workflows before scaling up.

The Facilitator's implementation strategies resulted in a shift in staff perceptions about the usefulness of PROMs, their knowledge about PROMs and how they could use them practically to improve their clinical care. Interestingly, whilst nursing knowledge and confidence improved markedly postimplementation, allied health professionals reported somewhat lower knowledge and confidence post-implementation. Whilst the reason for this is unclear, the very high selfreported knowledge and confidence pre-implementation may have reflected lack of experience applying PROMs within this complex patient group, with more conservative estimates after experiencing the PROM implementation. Additionally, the doctors and nurses may have had more exposure to the PROMs which may have increased their knowledge and confidence. Staff engagement with PROMs has been identified as essential for implementation success [29].

Staff surveys showed that the acceptability of PROMs increased from pre- to post-implementation. The construct of acceptability has been described in the literature as dynamic and changing as Recipients experience an innovation/intervention and gain a deeper understanding of "what" is involved [4], and this matched our experience in the case study. In the post-implementation survey sample, the perceived "usefulness" (relative advantage) of PROMs increased as clinicians started to report that they understood better the value of PROMS and reduced barriers to PROMs use $[4,21,46]$.

We also found that the collection of formative data in our case study was essential to enable successful facilitation. Staff responded to quantitative data of completion rates and staff acknowledgement rates with accompanying observational data, such as changes to workflows in response to events taking place in clinical areas. This use of data assisted in giving all Recipients a deeper understanding of the factors at play in the Context, and insight into the importance of the role staff have in the facilitating implementation.

Implementation science theories, such as i-PARIHS, can provide rich data to support and expand existing frameworks for PROMs. For example, Van der Wees and colleagues described a sequence of steps for choosing and implementing PROMs (goal setting, selecting PROs and PROMs, developing and testing of quality indicator(s), and implementing and evaluating the PROM(s) and indicator(s)) [8]. Our case study shows that it may be worthwhile to combine
PROM-specific frameworks with implementation science theories to better characterise the ways in which PROM attributes (e.g. validity and reliability) may interact with the implementation process. Implementation science theories systematise barriers so they can be linked to the broader implementation literature [47], directly link barriers to evidence-based support strategies [48] and provide a way to examine contextual factors influencing implementation (e.g. organisational readiness to change and clinic culture) [48].

Whether implementation is successful or not, there is much to learn when it is systematically described, and this knowledge is disseminated [49].

\section{Limitations}

We recognise some limitations with the evaluation. Data used to inform the analysis of the i-PARIHS constructs included observations collected by the Facilitator (NR) in site journals and field notes. This may be biased both by her perspective as a clinical trial nurse within the study context, and as the Facilitator, and are intrinsically subjective judgements. There is a balance in an implementation evaluation between the deep knowledge of context available to an internal Facilitator, and the risk of bias inherent in holding this tacit knowledge. Mechanisms to increase objectivity included contemporaneous notes, triangulation of data sources, reflexive monitoring with a research team, including members internal and external to the Context, and regular reflection with an expert Facilitator external to the project team. Staff surveys were anonymous, and used as cross-sectional data, and it is unknown where there is some overlap between respondents at both time points. The exact numbers of staff surveys distributed to each professional group was not able to be accurately captured, only the total number distributed due to the non-identifiable approach to data collection.

The detailed description of the Recipients and Context highlights the importance of local factors for implementation success, which means that findings may not be generalisable to other clinics or settings. However, these findings could inform approaches to implementation in other settings as the enablers and barriers had consistency with the findings of the initial scoping review [14].

\section{Conclusions}

The i-PARIHS framework was useful to systematically inform and iteratively plan the implementation of PROMs into routine oncology care. The four core i-PARIHS constructs (Facilitation, Recipients, Innovation and Context) all needed to be addressed to allow successful implementation, 
with Facilitation having a particularly important role moderating the other domains of Recipients, Innovation and Context. In this study, the PROMs intervention was acceptable to many staff, but complex barriers mean that sustainability may not have been achieved, and teams may struggle to maintain PROMs once the facilitation role has been withdrawn. Further research into efficient implementation and sustainability of PROMs is required to ensure lasting benefits.

Acknowledgements NR had support from a RBWH Post-graduate scholarship and QUT APA scholarship. Equipment and IT support during the study were funded by a Royal Brisbane and Women's Hospital Foundation Susan Sudak Day to End Cancers Equipment Grant and a Royal Brisbane and Women's Hospital Cancer Care Services General Practice Partners Grant.

\section{Compliance with ethical standards}

Conflict of interest All the authors declare that they have no conflict of interest to declare.

Ethical approval This implementation study underwent full Human Research and Ethics Committee (HREC) Approval (Approval number HREC/17/QRBW/416). Pre-implementation and post-implementation surveys were approved as a separate study (HREC/16/QRBW/100).

Open Access This article is licensed under a Creative Commons Attribution 4.0 International License, which permits use, sharing, adaptation, distribution and reproduction in any medium or format, as long as you give appropriate credit to the original author(s) and the source, provide a link to the Creative Commons licence, and indicate if changes were made. The images or other third party material in this article are included in the article's Creative Commons licence, unless indicated otherwise in a credit line to the material. If material is not included in the article's Creative Commons licence and your intended use is not permitted by statutory regulation or exceeds the permitted use, you will need to obtain permission directly from the copyright holder. To view a copy of this licence, visit http://creativecommons.org/licenses/by/4.0/.

\section{References}

1. Basch, E. B., Deal, A. M., Kris, M. G., Scher, H. I., Hudis, C. A., Sabbatini, P., et al. (2016). Symptom monitoring with patientreported outcomes during routine cancer treatment: A randomised controlled trial. Journal of Clinical Oncology, 34(6), 557-565.

2. Denis, F., Lethrosne, C., Pourel, N., Molinier, O., Pointreau, Y., Domont, J., et al. (2017). Randomized trial comparing a webmediated follow-up with routine surveillance in lung cancer patients. Journal National Cancer Institute, 109(9), 1-8.

3. Velikova, G., Booth, L., Smith, A. B., Brown, P. M., Lynch, P., Brown, J. M., \& Selby, P. J. (2004). Measuring quality of life in routine oncology practice improves communication and patient well-being: A randomised controlled trial. Journal of Clinical Oncology, 22(4), 714-723.

4. Proctor, E., Silmere, H., Raghavan, R., Hovmand, P., Aarons, G., Bunger, A., et al. (2011). Outcomes for implementation research: Conceptual distinctions, measurement challenges, and research agenda. Administration Policy Mental Health, 38, 65-76.
5. Stover, A. M., Stricker, C. T., Hammelef, K., Henson, S., Carr, P., Jansen, J., et al. (2019). Using stakeholder engagement to overcome barriers to implementing Patient- Reported Outcomes (PROs) in cancer care delivery: Approaches from three prospective studies. "PRO-Cision" Medicine Toolkit. Medical Care, 55(7), S92-S97.

6. Jacobsen, P. B., \& Lee, M. (2015). Integrating psychosocial care into routine cancer care. Cancer Control, 22, 442-449.

7. Croot, L., O'Cathain, A., Sworn, K., Yardley, L., Turner, K., Duncan, E., \& Hoddinott, P. (2019). Developing intervention to improve health: A systematic review of international practice between 2015 and 2016. Pilot and Feasibility Studies, 5, 127.

8. Van der Wees, P. J., Verkerk, E. W., Verbiest, M. E. A., Zuidgeest, M., Bakker, C., Braspenning, J., et al. (2019). Development of a framework with tools to support the selection and implementation of patient-reported outcome measures. Journal of Patient Reported Outcomes. https://doi.org/10.1186/s4168 7-019-0171-9

9. Rodin, G. (2018). From evidence to implementation: A global challenge for psychosocial oncology. Psychooncology, 27, 2310-2316.

10. Lee, S. J. C., Clark, M. A., Cox, J. V., Needles, B. M., Seigel, C., \& Balasubramanian, B. A. (2016). Achieving coordinated care for patients with complex cases of cancer: A multiteam system approach. Journal of Oncology Practice, 12(11), 1029-1038.

11. Reeves, S., Perrier, L., Goldman, J., Freeth, D., \& Zwarenstein, M. (2013). Interprofessional education: Effects on professional practice and healthcare outcomes (update). Cochrane Database Systematic Reviews, 3, 2213.

12. Rankin, N., Butow, P. N., Hack, T., Shaw, J. M., Shepherd, H., Ugalde, A., \& Sales, A. (2019). An implementation science primer for psycho-oncology: Translating robust evidence into practice. Journal of Psychosocial Oncology Research and Practice, 1(3), 14.

13. Aoun, S., Deas, K., Toye, C., Ewing, E., Grande, G., \& Stajduhar, K. (2015). Supporting family caregivers to identify their own needs in end-of-life care: Qualitative findings from a stepped wedge cluster trial. Palliative Medicine, 29(6), 392-400.

14. Roberts, N. A., Janda, M., Alexander, K., \& Wyld, D. (2017). Patient reported outcomes (PROs) in clinical practice: A scoping review of reviews, systematic review and meta-analysis. PsychoOncology, 26(3), 73.

15. Diffin, J., Ewing, G., Harvey, G., \& Grande, G. (2018). Facilitating successful implementation of a person-centred intervention to support family carers within palliative care: A qualitative study of the Carer Support Needs Assessment Tool (CSNAT) intervention. BMC Palliative Care, 17, 129.

16. Kislov, R., Pope, C., Martin, G. P., \& Wilson, P. (2019). Harnessing the power of theorising in implementation science. Implementation Science, 14, 103.

17. Stover, A. M., \& Basch, E. M. (2016). Implementing symptom questionnaires into oncology workflow. Journal of Oncology Practice, 12(10), 859-862.

18. Stover, A. M., Irwin, D., Chen, R. C., Chera, B. S., Mayer, D. K., Muss, H., et al. (2015). Integrating real-time, electronic patientreported measures into cancer care: Cancer patients' and clinicians' perceptions of acceptability and value. eGEMs Generating Evidence and Methods to Improve Patient Outcomes, 2(1), 23.

19. Craig, P., \& Petticrew, M. (2013). Developing and evaluating complex interventions: Reflections on the 2008 MRC guidance. International Journal of Nursing Studies, 50, 585-592.

20. Dueck, A. C., Mendoza, T. R., Mitchell, S. A., Reeve, B., Castro, K., Rogak, L., et al. (2015). Validity and reliability of the US national cancer institute's patient-reported outcomes version of the common terminology criteria for adverse events (PROCTCAE). JAMA Oncology, 1(8), 1051-1059. 
21. Harvey, G., \& Kitson, A. (2015). Implementing evidence-based practice in healthcare: A facilitation guide. London: Routledge.

22. Nilsen, P. (2015). Making sense of implementation theories, models and frameworks. Implementation Science, 10(53), 1-13.

23. Lynch, E., Mudge, A., Knowles, S., Kitson, A., Hunter, S. C., \& Harvey, G. (2018). "There is nothing so practical as a good theory": A pragmatic guide for selecting theoretical approaches for implementation projects. BMC Health Services Research, 18(857), 1-11.

24. Wang, A., Pollack, T., Kadziel, L. A., Ross, S., McHugh, M., Jordan, N., \& Kho, A. (2018). Impact of practice facilitation in primary care on chronic disease care processes and outcomes: A systematic review. Journal of General Internal Medicine, 33(11), 1968-1977.

25. Antunes, B., Harding, R., \& Higginson, I. J. (2014). Implementing patient-reported outcome measures in palliative care clinical practice: A systematic review of facilitators and barriers. Palliative Medicine, 28(2), 158-175.

26. Santana, M. J., Haverman, L., \& Absolom, K. (2015). Training clinicians on how to use patient-reported outcome measures in routine clinical practice. Quality of Life Research, 24(7), 1707-1718.

27. Foster, A., Croot, L., Brazier, J., Harris, J., \& O'Cathain, A. (2019). The facilitators and barriers to implementing patient reported outcome measures in organisations delivering health related services: A systematic review of reviews. Journal of Patient-Reported Outcomes, 2(46), 1-16.

28. Greenhalgh, J. (2009). The applications of PROs in clinical practice: What are they, do they work, and why? Quality of Life Research, 18, 115-123.

29. Nic Giolla Easpaig, B., Tran, Y., Bierbaum, M., Arnolda, G., Delaney, G. P., Liauw, W., et al. (2020). What are the attitudes of health professionals regarding patient reported outcome measures (PROMs) in oncology practice? A mixed methods synthesis of the qualitative evidence. BMC Health Services Research, 20(102), $1-24$.

30. Harvey, G., \& Kitson, A. (2016). PARIHS revisited: from heuristic to integrated framework for the successful implementation of knowledge into practice. Implementation Science, 11(33), 1-13.

31. Baskerville, N. B., Liddy, C., \& Hogg, W. (2012). Systematic review and meta-analysis of practice facilitation within primary care settings. Annals Family Medicine, 10(1), 63-74.

32. Parchman, M. L., Noel, P. H., Culler, S. D., Lanham, H., Leykum, L., Romero, R., \& Palmer, R. (2013). A randomized trial of practice facilitation to improve the delivery of chronic illness care in primary care: Initial and sustained effects. Implementation Science, 8, 93.

33. Klassen, A. C., Creswell, J., Plano Clark, V. L., Smith, K. C., \& Meissner, H. I. (2012). Best practise in mixed methods for quality of life research. Quality Life Research, 21(3), 377-380.

34. Ring, L., Gross, C. R., \& McColl, E. (2010). Putting the text back into context: Toward increased use of mixed methods for quality of life research. Quality of Life Research, 19(5), 613-615.

35. Roberts, N. A., Mudge, A., Alexander, K., Wyld, D., \& Janda, M. (2018). The iPROMOS protocol: A stepped-wedge study implement routine patient reported outcomes in a medical oncology outpatient setting. BMJ Open., 9, e027046.

36. Miles, M. B., Huberman, A. M., \& Saldana, J. (2010). Qualitative data analysis: A methods sourcebook (3rd ed.). Sage: London.

37. Rouette, J., Blazeby, J., King, M., Calvert, M., Peng, Y., Meyer, R. M., et al. (2015). Integrating health-related quality of life findings from randomized trials into practice: An international study of oncologists' perspectives. Quality of Life Research, 24, 1317-1325.

38. Mitchell, S. A., \& Chambers, D. A. (2017). Leveraging implementation science to improve cancer care delivery and patient outcomes. Journal of Oncology Practice, 13(8), 523-530.

39. Kirchner, J., Edlund, C. N., Henderson, K., Daily, L., Parker, L. E., \& Fortney, J. C. (2010). Using a multi-level approach to implement a primary care mental health $(\mathrm{PCMH})$ program. Families, Systems, \& Health, 28(2), 161-174.

40. Harvey, G., McCormack, B., Kitson, A., Lynch, E., \& Titchen, A. (2018). Designing and implementing two facilitation interventions within the 'Facilitating Implementation of Research Evidence (FIRE) study: A qualitative analysis from an external facilitators perspective. Implementation Science, 13(141), 1-14.

41. Stetler, C. B., Damschroder, L. J., Helfrich, C. D., \& Hagedorn, H. J. (2011). A guide for applying a revised version of the PARIHS framework for implementation. Implementation Science, 6(99), $1-10$.

42. Powell, B. J., Beidas, R. S., Lewis, C. C., Aarons, G. A., McMillen, J. C., Proctor, E. K., \& Mandell, D. S. (2017). Methods to improve the selection and tailoring of implementation strategies. Journal Behavioural Health Services Research, 44(2), 177-194.

43. Kislov, R., Humphreys, J., \& Harvey, G. (2017). How do managerial techniques evolve over time? The distortion of "facilitation" in healthcare service improvement. Public Management Review, 19(8), 1165-1183.

44. Kotronoulas, G., Papadopoulou, C., MacNicol, L., Simpson, M. F., \& Maguire, R. (2017). Feasibility and acceptability of the use of patient-reported outcome measures (PROMs) in the delivery of nurse-led supportive care to people with colorectal cancer. European Journal of Oncology Nursing, 29, 115-124.

45. Greenhalgh, T., Robert, G., Macfarlane, F., Bate, P., \& Kyriakidou, O. (2004). Diffusion of innovations in service organizations: A systematic review and recommendations. Milbank Quarterly, 82(4), 581-629.

46. Hull, L., Goulding, L., Kadjesari, Z., Davis, R., Healey, A., Bakolls, I., \& Sevdalls, N. (2019). Designing high-quality implementation research: development, application, feasibility and preliminary evaluation of the implementation science research development (ImpRes) tool and guide. Implementation Science, $14,80$.

47. Powell, B. J., Waltz, T. J., Chinman, M. J., Damschroder, L. J., Smith, J. L., Matthieu, M. M., et al. (2015). A refined compilation of implementation strategies: Results from the Expert Recommendations for Implementing Change (ERIC) project. Implementation Science, 10(21), 1-14.

48. Stover A. M., Haverman L., van Oers H., Greenhalgh J., Potter C.M., on behalf of the ISOQOL PROMs/PREMs in clinical practice implementation science workgroup. (2020). Using an implementation science approach to implement and evaluate PatientReported Outcome Measures (PROM) initiatives in routine care settings. Quality of Life Research

49. Bauer, M. S., Damscroder, L., Hagedom, H., Smith, J., \& Kilbourne, A. M. (2015). An introduction to implementation science for the non-specialist. BMC Psychology, 3, 32 .

Publisher's Note Springer Nature remains neutral with regard to jurisdictional claims in published maps and institutional affiliations. 